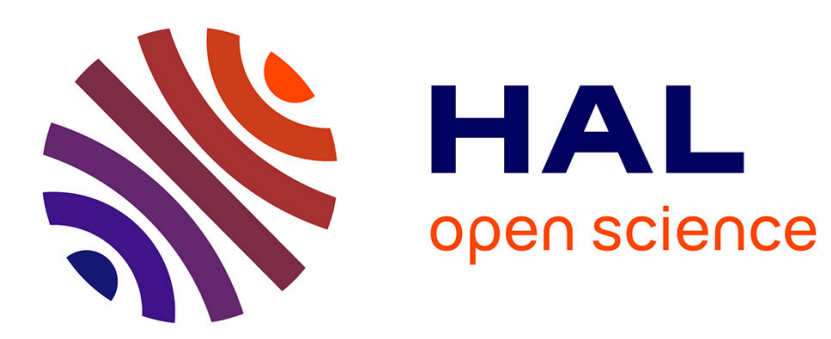

\title{
Fast Mutual Relative Localization of UAVs Using Ultraviolet LED Markers
}

\author{
Viktor Walter, Martin Saska, Antonio Franchi
}

\section{To cite this version:}

Viktor Walter, Martin Saska, Antonio Franchi. Fast Mutual Relative Localization of UAVs Using Ultraviolet LED Markers. International Conference on Unmanned Aircraft Systems (ICUAS 2018), Jun 2018, Dallas, TX, United States. hal-01814828

\section{HAL Id: hal-01814828 https://hal.laas.fr/hal-01814828}

Submitted on 13 Jun 2018

HAL is a multi-disciplinary open access archive for the deposit and dissemination of scientific research documents, whether they are published or not. The documents may come from teaching and research institutions in France or abroad, or from public or private research centers.
L'archive ouverte pluridisciplinaire HAL, est destinée au dépôt et à la diffusion de documents scientifiques de niveau recherche, publiés ou non, émanant des établissements d'enseignement et de recherche français ou étrangers, des laboratoires publics ou privés. 


\title{
Fast Mutual Relative Localization of UAVs using Ultraviolet LED Markers
}

\author{
Viktor Walter ${ }^{1}$, Martin Saska ${ }^{1}$, Antonio Franchi ${ }^{2}$
}

\begin{abstract}
This paper proposes a new methodology for outdoor mutual relative localization of UAVs equipped with active ultraviolet markers and a suitable camera with specialized bandpass filters. Mutual relative localization is a crucial tool for formation preservation, swarming and cooperative task completion in scenarios in which UAVs share working space in small relative distances. In most current systems of compact UAV swarms the localization of particular UAVs is based on the data obtained from motion capture systems for indoor experiments or on precise RTK-GNSS data outdoor. Such an external infrastructure is unavailable in most of real multiUAV applications and often cannot be pre-installed. To account for such situations, as well as to make the system more autonomous, reliance on onboard sensors only is desirable. In the proposed approach, we rely on ultraviolet LED markers, that emit light in frequencies that are less common in nature than the visible light or infrared radiation, especially in high intensities. Additionally, common camera sensors are sensitive to ultraviolet light, making the addition of a filter the only necessary modification, keeping the platform low-cost, which is one of the key requirements on swarm systems. This also allows for a smaller size of the markers to be sufficient, without burdening the processing resources. Thus the proposed system aspires to be an enabling technology for deployment of large swarms of possibly micro-scale aerial vehicles in real world conditions and without any dependency on an external infrastructure.
\end{abstract}

\section{INTRODUCTION}

Mutual relative localization of flying robots is indispensable in many real-world applications that require deployment of multiple Unmanned Aerial Vehicles (UAVs) sharing the same workspace in small relative mutual distances. Using compact multi-UAV systems brings numerous benefits including cooperative task completion, extension of reach of a single robot and distribution of capabilities into independent members. Moreover, several tasks that are not solvable by a single robot do exist and some of them were successfully solved by teams of UAVs developed by the Multi-robot Systems group at CTU in Prague ${ }^{3}$ by employing onboard visual mutual relative localization - see Fig. 1 a) and c) for examples. In this paper, we propose a novel robust method of infrastructure-independent relative localization for flights of multiple UAVs, applicable for outdoor environments, as

\footnotetext{
${ }^{1}$ Authors are with Faculty of Electrical Engineering Czech Technical University, Technicka 2, Prague, Czech Republic

\{viktor.walter|martin.saska\}@fel.cvut.cz

${ }^{2}$ Author is with LAAS-CNRS, Université de Toulouse, CNRS, Toulouse, France

antonio.franchielaas.fr

This research was partially supported by the ANR, Project ANR-17CE33-0007 MuRoPhen and by CTU grant no. SGS17/187/OHK3/3T/13, the Grant Agency of the Czech Republic under grant no. 17-16900Y

${ }^{3}$ https://mrs.felk. cvut.cz
}

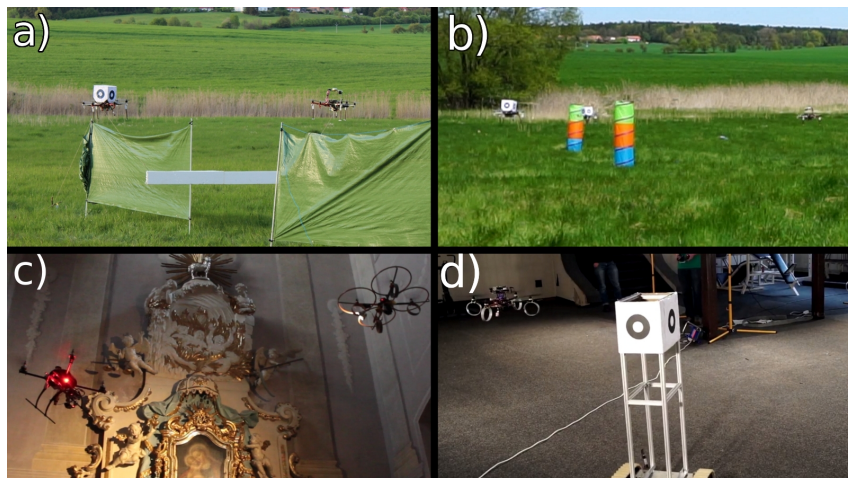

Fig. 1: Examples of applications of mutual relative localization developed by our group. a) Cooperative carrying of large objects, b) simultaneous flight through forest-like environments using bioinspired swarming rules, c) mapping of historical buildings by a formation of camera-carrying UAV and spotlight-carrying UAVs to obtain footage with fixed illumination angle d) heterogeneous formation of self-stabilised UAV-UGV.

well as the indoors. The method is based on the application of markers composed of ultraviolet LEDs on the UAVs, in addition to equipping the observer UAVs with cameras with fisheye lenses and specialized bandpass filters. The relative pose of the observed UAVs can then be retrieved easily from the image, where the markers are visible as bright spots on dark background that can be located with little processing. Our intention is to provide each swarm member with an as complete as possible information on the state of all UAVs in close proximity without any the of communication among the robots or with a base station. Fulfilling this goal requires direct sensing of mutual relative distances, information about the relative bearings and, if possible, also about their relative headings.

As can be seen in numerous examples of flocking of animals, such information can be effectively obtained through vision [1]. In our previous works on mutual localization [2], [3], passive markers in conjunction with an object detection algorithm have been used to achieve the same sensory ability. This vision-based system has been deployed in numerous indoor and outdoor experiments [4], [5], [6], where multiple drawbacks, such as strong lighting conditions dependency, large size of the markers, limited operational space and computational complexity have been identified. The most significant is the fact that the changing outdoor lighting conditions may prevent consistent recognition of the markers. The system proposed in this paper aims to alleviate these drawbacks and to further enlarge the application domain of UAV swarms. 


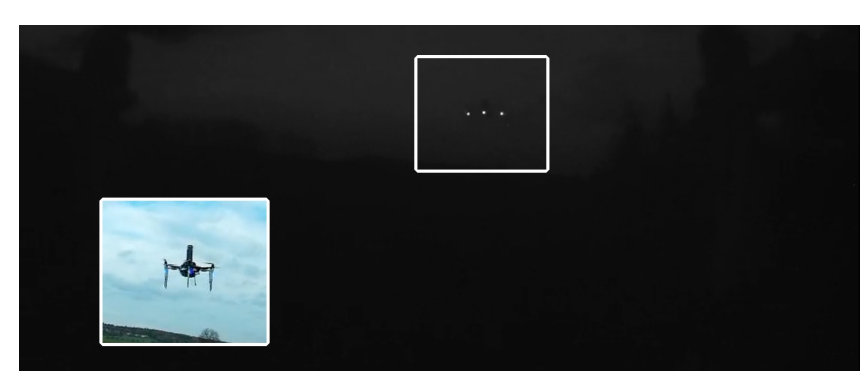

Fig. 2: Example of an image from the onboard camera with filter of a hexarotor UAV equipped with 6 markers on its arms. The picture was taken at noon, with the exposure rate set to $1000 \mu$ s. Lower left shows the same view from a conventional camera.

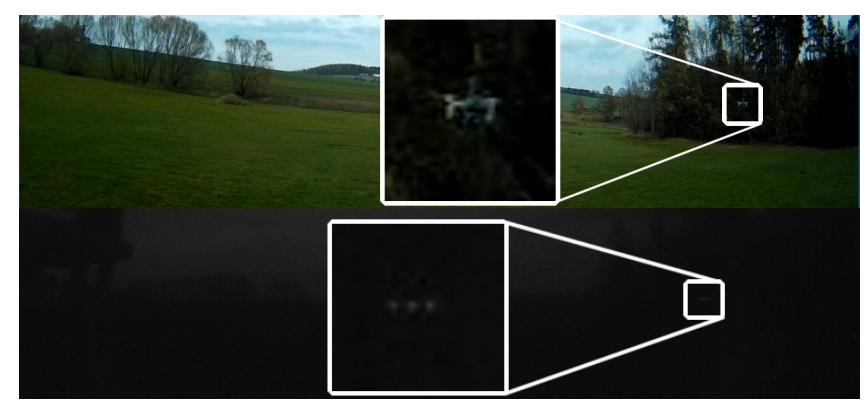

Fig. 3: An extreme case of long range and hard to separate background. In the visible spectrum the UAV is difficult to locate even by the human eye and today's popular CNN methods [7], while in UV the three markers are clearly visible as unique peaks in brightness and can easily be detected by the proposed system.

\section{A. State of the art and contributions}

Most of the multi-UAV experiments that require localization have been realized in laboratory conditions relying on an absolute measurement by a motion capture system $\left(\right.$ Optitrack $^{4}, \mathrm{VICON}^{5}$, etc.) $[8]$ and the relative pose measurement is emulated by calculating it from a source of absolute pose as a placeholder [9]. Outdoor cooperative flights [10], [11] tend to rely on GNSS (Global Navigation Satellite System) if a close proximity of robots is not required or on RTK-GNSS data (provided in our case by Tarsus BX305 ${ }^{6}$ ), which may achieve the precision of $\pm 10 \mathrm{~mm}$. Obviously, these approaches suffer from the necessity to pre-install an external infrastructure (motion capture cameras or RTK basestation) which precludes flights in environments that are unknown or difficult to reach by the operators themselves. Another problem is transition from outdoor to indoor environments, flight near elevated objects of larger volume buildings, rock formations, etc. - or flight in an unknown, cluttered or inaccessible enclosed environment. Additionally, continual reception of such external information requires wireless communication, which is subject to limited range and interference both from unrelated sources and by the units themselves in the case of a large swarm. This makes the

\footnotetext{
${ }^{4}$ http: // optitrack.com/

5 https://www.vicon.com/motion-capture/engineering

${ }^{6}$ https://cdn. shopify.com/s/files/1/0928/6900/

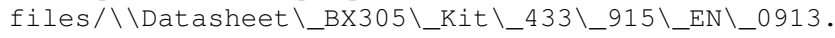
pdf
}

system difficult to scale up to larger number of units, which is the main idea of robotic swarms. Another challenge caused by the infrastructure dependence the task may easily be deliberately obstructed by interfering with the infrastructure, such as by introduction of artificial radio interference on key radio frequencies. In order to be reliable even in such circumstances, the units need to be able to independently avoid damage and complete their mission. If the UAVs are flying in a formation, they should be able to preserve it or keep their mutual distances within safe ranges, which is reliably enabled by the proposed approach.

Numerous principles of direct mutual localization can be found in literature. From a theoretical point of view the mutual localization problem in group of robots boils down to the (bearing) rigidity problem, see [12] and reference therein for an introduction to this concept. The multi-agent mutual localization problem has been also faced in the case in which measurements do not provide the identity of the measured robot, i.e., they are anonymous [9].

Relative localization of noise-emitting objects such as UAVs was successfully tested in [13]. This method, however, requires a large and highly specialized equipment, while providing only an approximate relative bearing of the target and being sensitive to acoustic environmental noise.

Another approach was used in [14], where point-clouds obtained from RGB-D cameras attached to each unit are aligned to obtain their relative poses. These sensors have severely limited range and field of view and the algorithm is computationally too complex for most onboard computers of lightweight UAVs. Similarly, [15] used the alignment of lines detected in images of the environment of the UAVs to establish their relative positions. Such an approach can be applied efficiently in an environment with multiple straight lines, such as in streets or offices, but not in natural environments where straight lines are rare. Both of the aforementioned approaches additionally require communication between the units, at fairly high bandwidths.

Numerous experimental solutions based on vision and mutual observation of UAV and UGVs equipped with known geometrical markers were tested [16], [17]. Our previous solution uses circular visual markers [2], [3] for mutual localization in small swarms of UAVs [5], [6] and in heterogeneous formations [4]. The main disadvantage of these methods is the sensitivity to light conditions, computational complexity and the physical dimensions of these markers. The large size of the markers (see fig. 1-a), needed for proper detection from reasonable distances and angle range leads to problems connected to aerodynamics and maintenance difficulties. In addition, these markers are highly susceptible to a partial occlusion that can prevent detection.

A basic and often used approach is to apply a simple, easy to segment color-based markers that work well in controlled light conditions of laboratory environments [18], [19]. While in a laboratory it is easy to apply active or passive markers of a color that we ensure is not otherwise present in the environment, this is seemingly unfeasible in outdoor conditions, since in nature as well as in urban scenarios, all 
colors of the visible spectrum are naturally present.

The closest approach to the proposed system can be found in [20], where UAV localization through infrared lights blinking in the order of kilohertz attached to helicopters is implemented. Such frequency allows for the markers tracked even with aggressive flight maneuvers. This was achieved by using an event-based camera. The solution in [20] was only tested in indoor environments, with the camera being statically placed in the room, instead of onboard of a UAV. The use of a heavy and expensive specialized camera makes it unsuitable for outdoor onboard deployment. Additionally, the low resolution of contemporary event-based cameras decrease the precision and the range of detection.

The system presented here is based on the observation that while the colors of the visible spectrum, as well as a wide band of the infrared wavelengths, are present with relatively high intensity in normal sunlight, the ultraviolet light is significantly less intense. This means that in the UV spectrum the natural environments are dark and when an object bright in the ultraviolet is observed, it is likely to be artificial. Exploiting this fact does not even require a new specialized sensor, since common monochromatic digital cameras tend to be receptive to near ultraviolet frequencies, and need only be modified with a proper band-pass filter. The UV light can then be used as a robust and easy detect feature. The only false positives in detection being the sun itself and its specular reflections, while even most of artificial light sources emit little to none UV radiation.

To sum up the contribution of the paper related to the current literature, we emphasize that the proposed solution combines knowledge gained during hundreds of flights with multiple closely cooperating UAVs in realistic indoor and outdoor conditions using different state-of-the-art localization systems and a theoretical analyses of sources of disturbance that lead to false positive detections in common workspaces of UAV systems. Based on this data we propose the HW design of a system made of multiple onboard UV light sources that reliably provides the required information (distance and bearing of neighbouring vehicles) in all possible configurations of the team. In comparison with state-of-the-art solutions, our solution presents better reliability w.r.t different weather conditions and precision, while significantly reducing size and weight of the overall system and the required computational power. We provide two approaches for mutual localization using this HW setup. The first minimalistic approach uses a single camera and a single UV LED on each UAV to provide bearing information and a rough estimation of the relative distance. This method together with the work [21], where we have shown that such a sensory information (even anonymous) is sufficient for reliable coherent swarming, provides a complex solution for deployment of large swarms of micro aerial vehicles. The scalability is shown in [21] by a surprising observation that the swarm stability and coherence increases with number of swarm members even with such a limited sensory input. The second approach presented in this paper exploits possibility of using multiple LEDs onboard of UAVs to increase pre-

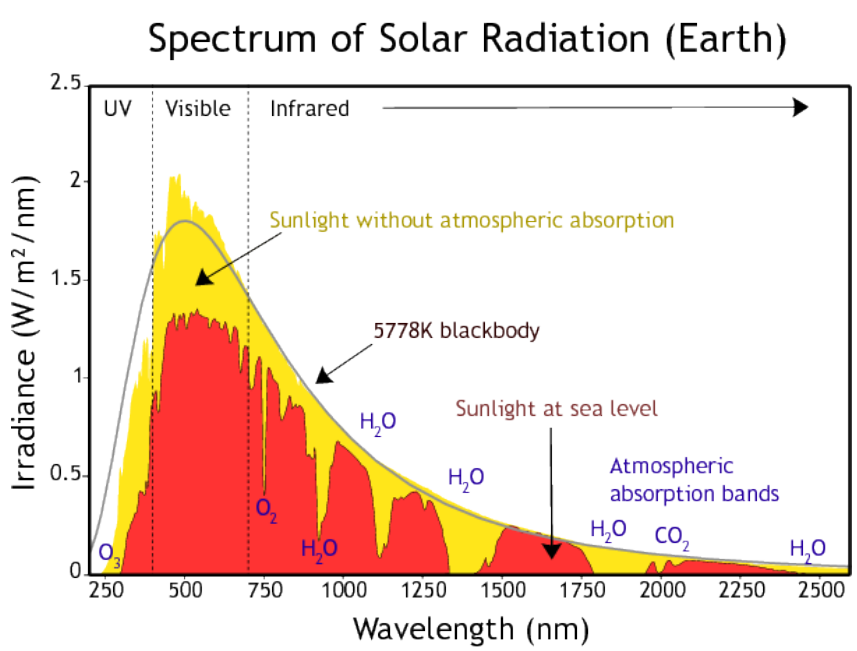

Fig. 4: The spectrum of solar radiation in wavelengths near the visible spectrum. Notice the rapid decrease in irradiance in the UV region. ${ }^{7}$

cision of the distance measurement and operational space. In fact, this approach exploits full size of the UAVs, putting the LEDs as far apart as possible, which increases baseline used for the distance estimation, resulting in higher precision, in comparison with passive markers that are always significantly smaller that the UAV itself.

The paper is structured as follows. Section II deals with the theoretical background applied in the design of the system. Section III comprises the overview of the hardware used. Section IV explains the methodology used in estimating the mutual location of the target (neighbouring UAV) as well as the system identification. Finally, section V summarizes the results of experimental testing of the system.

\section{THEORETICAL BACKGROUND}

\section{A. The ultraviolet markers and sensors}

Using UV light for mutual localization is an obvious approach for deployment of swarms in real outdoor environments, as it was the case in our experiments, but not in typical laboratory experiments [8], [22]. The solar radiation approximates the blackbody radiation, with peak intensity centered on the visible light (see figure 4). This means that the shape of the intensity to wavelength characteristic is asymmetric w.r.t. the wavelength. The intensity decays considerably slower with growing wavelengths than it does with diminishing wavelength. Ultraviolet parts of sunlight are therefore significantly less intense than the visible light and the infrared, even relatively close to the visible spectrum. We can exploit this observation to apply active markers that can be easily distinguished from the multicolored natural backgrounds purely based on the intensity. In the implementation, this requires physical band-pass filtering tuned to the ultraviolet wavelengths of the markers.

\footnotetext{
${ }^{7}$ https://commons.wikimedia.org/w/index.php?title= File:Solar_spectrum_ita.svg\&oldid=261911890
} 
A gray-scale camera is more suitable for this task since it is less selective w.r.t. the wavelength than RGB cameras and is thus more sensitive to light outside of the visible spectrum. Note, that while the sun does still emit considerable amounts of UV radiation, this radiation is spread across a range of wavelengths and most of it is filtered out by the atmosphere. Additionally, it does not reflect well on solid natural surfaces. With low enough exposure rate of the camera, the only objects that will locally saturate the resulting image to white will be the markers, directly observed sun and some of its specular reflections. The appropriate exposure rate depends on the required maximal detection distance. Our tests have shown that the UV light refracted through the atmosphere and reflected from matte surfaces is normally too dim to prevent the detection. The image of the sun and its reflections should be a minor issue which can be accounted for by using knowledge of the size or other specific characteristics of the spots caused by the sun or the knowledge of sun position based on current place and time. The possibility to set the exposure so that the markers will create saturated white spots on dark background can be used to binarize the image using simple static thresholding, as opposed to the more computationally demanding adaptive thresholding needed for arbitrary lighting conditions. The image spots caused by artificial light sources will be saturated regardless of the time of the day.

In order for this system to be suitable for mutual localization of multiple UAVs, the camera has to be able to observe the surroundings in a large field of view. This can be achieved by using a fisheye lens. A potential drawback that has to be addressed is the high rate of decay of UV light in common types of glass, proportional to the specific frequency. In our tests, this has limited the wavelength of the UV light that we could use. Light sources radiating at $365 \mathrm{~nm}$ were suppressed to the extent that they were mostly invisible to the camera, while light sources with wavelengths of $395 \mathrm{~nm}$ were clearly visible. Specialized lenses permeable to high-frequency UV that also have wide FoV and are portable could not be found on the market at the current time. Moreover, the infrared filter applied to the lenses by the manufacturer also blocks out UV light. A shorter wavelength would allow for better filtering of of the sunlight. Despite this, the $395 \mathrm{~nm}$ light sources and filters proved to be sufficient in our experiments.

\section{HARDWARE OVERVIEW}

The camera used in our experiments is based on the Matrix Vision mvBlueFOX-MLC200wG sensor, equipped with the Sunnex DSL215 fisheye lens and Midopt BP365-R6 ultraviolet bandpass filter.

The mvBlueFOX-MLC200wG (figure 5-c) is a greyscale CMOS sensor with a resolution of $752 \times 480$ pixels, maximum frame-rate of 93 without binning and quantum efficiency at $395 \mathrm{~nm}$ of $\approx 34 \% 8$ In our experiments we were able to achieve a maximum frame-rate of $70 \mathrm{~Hz}$ with the exposure rate set to $1000 \mu \mathrm{s}$. The DSL215 is a fisheye lens

\footnotetext{
${ }^{8}$ https: / / www matrix-vision. com/ \\USB2. 0-single-board-camera-mvbluefox-mlc.html
}

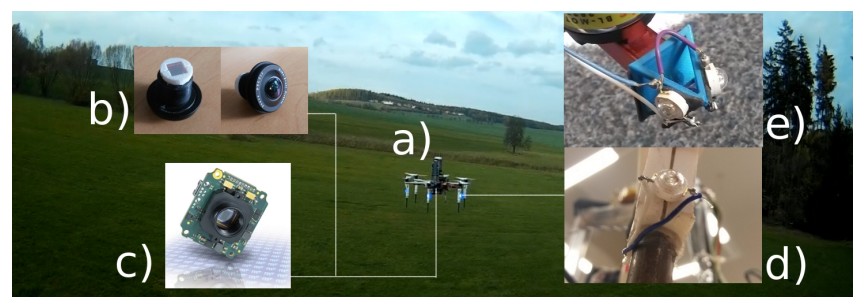

Fig. 5: Summary of the proposed system components. The UAV (a) is equipped by a mvBlueFOX-MLC200wG camera sensor (c) with DSL215 lens with BP365-R6 bandpass filter that allows it to observe and localize ultraviolet LED-based markers (d) or (e)

with the maximal FoV of $185^{\circ}$. This value, however only applies to the horizontal field of view with the $M L C 200 w G$ sensor. ${ }^{9}$ The BP365-R6 is a miniature interference-based optical bandpass filter for ultraviolet imaging ${ }^{10}$. The custom size of $6 \times 6 \times 1 \mathrm{~mm}$ allowed us to attach it between the lens and the sensor so that the whole image is covered, as seen in figure 5-b. For the markers, we have selected the ProLight PM2B-1LLE, (figure 5-d) which is a high power ultraviolet LED, with the maximum of emission centered on the wavelength of $395 \mathrm{~nm}$ and with Lambertian radiation pattern. ${ }^{11}$

This beacon-sensor system is small, lightweight and relatively affordable, and thus ideal for deployment with small UAVs.

\section{METHOD DESCRIPTION}

\section{A. System identification}

1) Camera calibration: In order to convert the image positions of the detected spots corresponding to the markers on the UAVs into relative bearing vectors, it was necessary to perform geometric calibration of the camera. We have selected the model described in [23], suitable for cameras with wide FoV, that translates pixel positions directly into bearing vectors.

The parameters of the camera projection are affected by the different index of refraction of UV light compared to the visible light, as well as by small eccentricities of the lens mount. To account for these factors we calibrated the camera with the band-pass filter on and the lens attached in the final position. For the chessboard-type calibration pattern to be fully visible, the pattern had to be illuminated by a UV light source, with the exposure rate and threshold manually adjusted for the different angles of view, some parts of the pattern became overbright or overly dark depending on the angle of reflection. After obtaining the images, the calibration was done semi-automatically using the OCamCalib toolbox 12. The toolbox itself provides a method of converting pixel position into a unit bearing vectors of the markers, here denoted $c 2 w$ and the reverse function $w 2 c$.

\footnotetext{
${ }^{9}$ http: //www.optics-online.com/ooL/dsl/dsl215.pdf

10 http: //midopt.com/filters/bp365/

${ }^{11}$ http: / / datasheet.octopart.com/\। PM2B-1LLE-Prolight-datasheet-41916849.pdf

12 https://sites.google.com/site/scarabotix/ ocamcalib-toolbox, [24]
} 
The angle $\phi$ between the optical axis of the camera and an optical ray corresponding to a point in the image was modeled as a linear function of the distance $r$ of the point from the optical center in pixels. The slope of the function was measured to be

$$
k=\phi / r=3.7673 \times 10^{-3} \operatorname{rad~px}^{-1} .
$$

2) Spot size: An interesting imaging effect that can be exploited in this setup is the blooming effect of the camera. The markers used here are small LEDs, which with an idealized camera each LED would be projected into a single point on the sensor resulting in a single bright pixel. With real-world cameras, these markers are shown in the image as spots of sizes depending on the distance. This effect occurs due to a combination of monochromatic optical aberrations of the lens and of the finite capacity of the CMOS elements in the sensor causing excess charge being spilled over to the surrounding pixels. The exact analysis of the nature and relative impacts of these phenomena is beyond the scope of this paper.

The sizes of the spots can, however, be analyzed w.r.t. the distance of the marker (see figure 6) and thus used to give a rough estimate of the distance of the marker. Since the geometry of these spots depends on many unknown variables, and due to the finite resolution of the camera, the true position of the ray incidence within such a spot is ambiguous. The spots shrink down to the size of a single pixel at a certain distance from the camera, depending on the resolution, exposure rate, type of sensor and the radiation intensity of the marker in the direction of the camera. In order to preserve high output rate and ease of processing, we store the sizes of these spots in terms the number of pixels $S$ after thresholding. To represent the position of the spot, we store the $x$ and $y$ image coordinates of the middle pixel in order of contour filling.

Since the tend to be slightly blurry around their edges, the size of the spots after binarization depends on the selected threshold.

While the estimation of distance based on such an imprecise information is not ideal, it can inform of a neighbor breaching certain safety radii or leaving the minimal mutual distance. With the algorithm presented in [21] and the proposed approach, we can achieve coherent swarming using a minimal required mutual localization setup consisting of one simple camera and LED per unit (total mass of $50 \mathrm{~g}$ ), which opens new perspectives in use of micro aerial vehicles (MAVs).

Additionally, knowing the characteristics of the spot sizes $S(l)$ w.r.t. the distance from the camera $l$ is useful for establishing margins of error for measurements based on the estimated bearing vectors. For this purpose, we can select a function $S_{\max }(l)$ such that the values will lay under it. These were measured in experiments and can be seen in the next sections.

\section{B. Directional vector estimation}

Measuring the relative bearing between UAVs is one of the main requirements to let them stably fly in swarms with

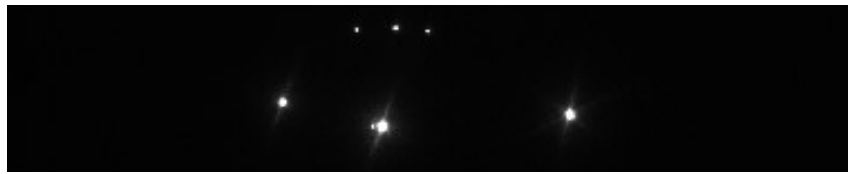

Fig. 6: Relative difference in sizes of spots generated by markers attached to quadrotor UAVs approximately $2 \mathrm{~m}$ (lower triplet) and $5 \mathrm{~m}$ (upper triplet) away from the camera.

a predefined shape. However, it is not always necessary for each UAV to measure all the relative bearings w.r.t. all the other members. Indeed, Franchi et al. [22], [9] have shown that a stable, controllable swarm with a predetermined shape can be achieved by resorting only to a certain minimum number of directed observations of relative bearings between the swarm members with known identities. It was also demonstrated [25] that it is possible to reconstruct the shape of the swarm purely based on relative bearings of the unit even when the identities of the observed neighbors are unknown. With our system, such control algorithm will be applicable not only to units with limited processing and data transfer capabilities but also to outdoor applications. Since we have already calibrated the camera, we can convert a pixel position $m_{i}$ to the relative bearing vector $v_{i}$ towards a single marker as

$$
v_{i}=c 2 w\left(m_{i}\right)
$$

In reality the pixel position $m_{i}$ corresponds to a range of bearing vectors that are projected to the same CMOS element, while the vector $v_{i}$ corresponds to the center of that range. In the worst case, the spot is a linear chain and the true bearing vector corresponds to the farthest point of the pixel located the farthest away from the pixel we have stored to represent the spot position. The calculated relative bearing vector $v_{i}$ can, therefore, differ from the true bearing vector $v_{b}$ at most by the angle

$$
\epsilon=k\left(S_{\max }(l)-\frac{1}{2}\right) .
$$

C. Distance estimation based on image distance of two markers

The distance of an object in an image can be estimated based on the distance of two points $M_{1}$ and $M_{2}$ on the object (two LEDs of known relative position onboard of the UAV) and the angle $\alpha$ they form with the camera origin $C$. This requires for the camera to be calibrated, and is only applicable if we have a reasonable assumption on the angle between $\overleftrightarrow{M_{1} M_{2}}$ and the direction towards the camera. For this method to be used for UAV localization, there needs to be at least a pair of visible markers. These two markers should not deviate too much from perpendicular alignment w.r.t. the direction towards the camera.

In order to limit the dependence of the precision of the distance estimation on the relative orientation of the observed UAV, the markers should be placed along a circle centered on the UAV local frame of reference, with equal distances between adjacent markers. Additionally, these markers should be spaced as far apart as possible, while still allowing 

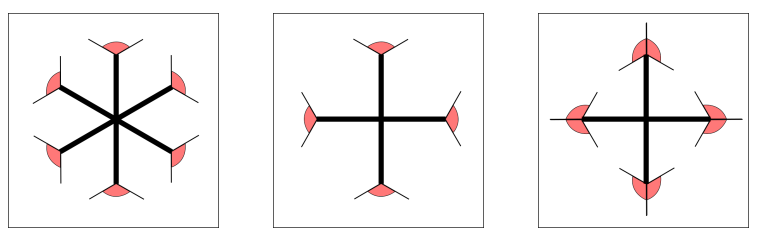

Fig. 7: Allignments of LED markers for a hexarotor, and a quadrotor. In the center layout, only a single marker would be visible from some directions, as opposed to the rightmost layout, with markers consisting of two LEDs at the mutual angle of $120^{\circ}$.

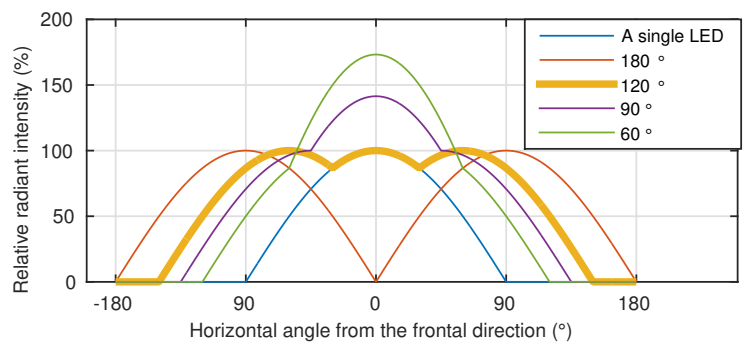

Fig. 8: The relative radiant intensities w.r.t. the direction for two combined, symmetrically aligned ideal Lambertian radiators. With the mutual angle being $120^{\circ}$ the intensity in the central direction is the same as for a single radiator aimed in that direction.

at least a single adjacent pair to be visible from every direcion. For multirotor UAVs with a star-like layout, with the propellers attached to equally spaced arms extending from the center, the best choice is to attach these markers at the ends of these arms. In other cases, they can be attached to a protective cage or on specific additional components, to ensure radial symmetry. For a hexarotor UAV with equal arm lengths and internal angles, which is our most common use-case, the markers, composed of a single LED each, are attached to the ends of the arms. In this case a single LED per arm is sufficient, since the intensity of radiation in a Lambertian radiator (such as our chosen LEDs) at the angle of $60^{\circ}$ will not drop below $50 \%$ of the intensity in the frontal direction. In a similar quadrotor UAV, the markers must be composed of symmetrically angled pair of LEDs to account for the negligible intensities of radiation in the perpendicular direction in a single LED (see figure 7 and 5-e).

We recommend for the angle between these two LEDs to be $120^{\circ}$ in order to get approximately the same radiation intensity in the direction away from the center as with a single centered LED (see figure 8).

For a hexarotor with such markers we can estimate the distance in a range defined by two borderline assumptions:

1) The observed pair is in perpendicular alignment w.r.t. the direction towards the camera - camera in position $C_{A}$ in figure 9

2) One marker of the pair is on the connecting line of the hexagonal frame - camera in position $C_{B}$ in figure 9.

Which situation is currently closer to being the case unknown. While hexarotor is used as an example, similar borderline assumptions can be defined for quadrotors, octarotors, etc.

Depending on whether we need to check for UAVs being

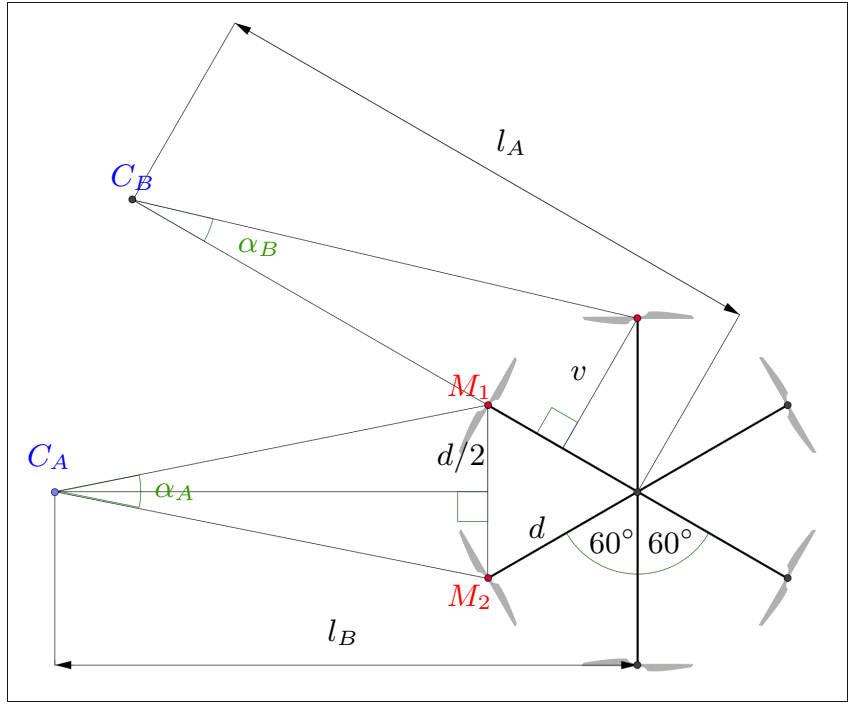

Fig. 9: Schematic of the two borderline algnments of a hexarotor and the camera. With the camera in position $C_{A}$, the observed pair is in perpendicular alignment w.r.t. the camera. In position $C_{B}$ the points are in the $30^{\circ}$ alignment w.r.t. the camera.

too close or too far away, we can select either calculation. In the case 1) the UAV is more likely to be closer than estimated, while in the case 2) it is more likely to be further away. In a swarming algorithm such as [21] the UAVs need to compare the distances of neighbors with two margins, the far limit and the near limit, between which the distance should be kept. In order to make such swarming more reliable, it makes sense to calculate with case 1) for the far limit and with case 2) for the near limit. This is also applicable for tasks such as cooperative carrying of large objects (see figure 1-a), where these limits are connected with the level of control over the object, collision avoidance and energy conservation.

The distance can be calculated from the triangle formed by the camera $C$ and the two markers $M_{1}$ and $M_{2}$. The parameter $d$ is distance between $M_{1}$ and $M_{2}$ and $\alpha$ is $\measuredangle M_{1} C M_{2}$. The relative bearing vectors from the camera towards points $M_{1}$ and $M_{2}$ are denoted $\mathbf{v}_{\mathbf{1}}$ and $\mathbf{v}_{\mathbf{2}}$.

$$
\alpha=\arccos \left(\mathbf{v}_{\mathbf{1}} \cdot \mathbf{v}_{\mathbf{2}}\right)=\arccos \left(c 2 w\left(\mathbf{m}_{\mathbf{1}}\right) \cdot c 2 w\left(\mathbf{m}_{\mathbf{2}}\right)\right)
$$

In the case 1 ), the distance $l_{A}$ to the UAV center can be expressed as

$$
l_{A}=\left(\frac{d}{2}\right) \cot \left(\frac{\alpha}{2}\right)+v,
$$

while in case 2), the distance $l_{B}$ can be expressed as

$$
l_{B}=v \cot (\alpha)+\left(\frac{d}{2}\right) .
$$

The symbol $v$ here stands for the height of an equilateral triangle with side $d$.

The intensity of radiation in a Lambertian light source, such as the one we are using is roughly proportional to the cosine of the angle from the axis. It therefore is more common to see only two of the six markers than three or four. 
To establish error margins for the two cases at ground truth distance $l$, we take the assumption that the wrong case was presumed, so that while calculating for case 1), the real situation corresponded to case 2) and vice-versa. If in the case 2) the calculation for the case 1) was used, the relation of the estimated distance to the real distance would be

$$
l_{\text {aerr }}=\left(\frac{d}{2}\right) \cot \left(\arctan \left(\frac{v}{l-\frac{d}{2}}\right) / 2\right)+v \text {. }
$$

In the converse case, the erroneous selection of calculation results in the estimate

$$
l_{\text {berr }}=v \cot \left(2 \arctan \left(\frac{\frac{d}{2}}{l-v}\right)\right)+\left(\frac{d}{2}\right) .
$$

These margins should be additionally expanded by the effects of finite resolution and spot size. Due to foreshortening, as the UAV retreats away from the camera, the change of mutual distance of the two markers in the image becomes less and less pronounced. This is more significant when the mutual distance of the images of the markers becomes comparable with the size of a pixel. As was the case in the directional vector estimation, the maximum angular error $\epsilon$ in the direction of the vector $\mathbf{v}_{\mathbf{1}}$ is equal to $k\left(S_{\max }(l)-\frac{1}{2}\right)$

The final maximal value of distance estimation in the first case is then:

$l_{\text {amax }}=\left(\frac{d}{2}\right) \cot \left(\frac{\arctan \left(\frac{v}{l-\frac{d}{2}}\right)-k\left(S_{\max }(l)-\frac{1}{2}\right)}{2}\right)+v$

The minimal value for this case is:

$l_{\text {amin }}=\left(\frac{d}{2}\right) \cot \left(\arctan \left(\frac{\frac{d}{2}}{l-v}\right)+k\left(S_{\max }(l)-\frac{1}{2}\right)\right)+v$

In the other case, where we presume that one of the spots in the observed pair corresponds to a marker on the line connecting the camera and the center of the UAV, the maxima and minima of the estimation can be expressed similarly:

$l_{b \max }=v \cot \left(\arctan \left(\frac{v}{l-\frac{d}{2}}\right)-k\left(S_{\max }(l)-\frac{1}{2}\right)\right)+\left(\frac{d}{2}\right)$

$l_{b \min }=v \cot \left(2 \arctan \left(\frac{\frac{d}{2}}{l-v}\right)+k\left(S_{\max }(l)-\frac{1}{2}\right)\right)+\left(\frac{d}{2}\right)$

\section{Full pose estimation}

For a more precise position estimation of a neighbor UAV that is more suitable for $3 \mathrm{D}$ environments and returns orientation as well, we might consider using a Perspectiven-Point method. This may prove quite challenging in implementation, due to the anonymity of the observed points and due to the diminished visibility of the markers not facing the camera. Additionally, these methods are computationally more complex, which may reduce the output rate. One way to increase the number simutaneously of visible points is to increase the number of the LEDs on the UAV. This may be done in two possible ways:

1) by composing each marker out of multiple LEDs, as can be seen in figure 5-e

2) by adding more single-LED markers for denser coverage.

Care should be taken with such a modification, since if the distribution of the markers is too dense then they will tend merging in the image into a single spot. The problem of anonymity can also be addressed this way, by constructing patterns that can be matched with a known template without ambiguity.

Another potentially effective approach under consideration is to encode information such as individual ID of a marker into blinking patterns.

\section{EXPERIMENTS}

\section{A. Distance estimation based on spot size}

To evaluate the relationship between the mutual distance and the size of the bright spot an Optitrack motion-capture system was used to record the ground-truth distance between the camera and a single LED in its view, while the size of the spot was being recorded simultaneously. This procedure was repeated for multiple exposure rates. The binarization threshold used for segmentation of the bright spots was set to 200 out of 255 , or $78.42 \%$ of the saturated brightness (for the characteristics, see figure 10). These tests seem to indicate, that the best exposure rate for detecting whether the neighbors are within a reasonable distance with this setup is $1000 \mathrm{~ms}$, when the spot shrank consistently to the size of a single pixel at $6.62 \mathrm{~m}$ while still sufficiently filtering out the ambient UV illumination. Therefore, a very simple and low-cost approach (only one camera and one LED is required) can be used for an effective collision avoidance as it provides sufficient safety distance. For example the safety distance used in Multi-robot Systems group in [26] was $5 \mathrm{~m}$. Within the range from $3.14 \mathrm{~m}$ to $6.62 \mathrm{~m}$ the occurence of spots with the size of 1 pixel become common. For distances smaller than $3.14 \mathrm{~m}$ the non-linear the characteristic can be used for a rough, but quantitative distance estimation in applications, where the UAVs have to operate within very close mutual distances. From the characteristic for the $1000 \mu$ s exposure rate (figure 11) we have derived the parameters of an approximating equation (13) in the form of a quadratic-hyperbolic function rounded to the nearest integer.

$$
S_{\max }=\left\lfloor 1.3398+\frac{31.4704}{(x-0.0154)^{2}}\right\rceil .
$$

A quadratic-hyperbolic function was selected because the number of saturated pixels from a single ray depends roughly on the overall energy that has been received by the sensor from the marker, which in turn is governed by the inverse square law.

A different exposure rate can be selected for tasks where closer or greater mutual distances are required (note the different single-pixel thresholds in figure 10). For example, 


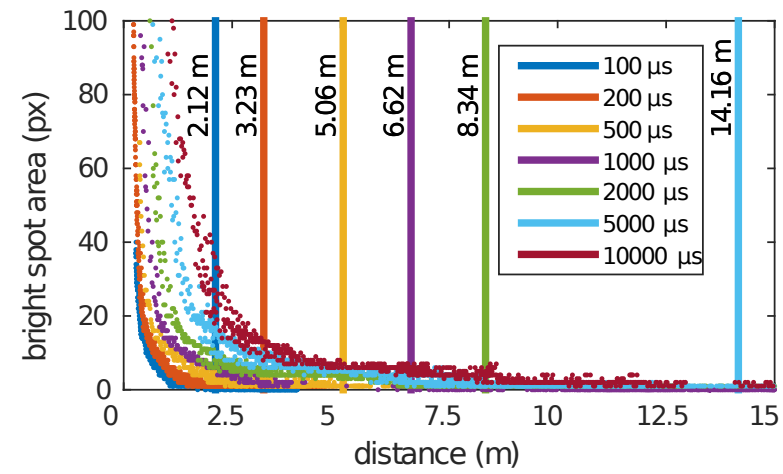

Fig. 10: Distance dependence of the bright spot size for multiple exposure rates. The vertical lines denote distances beyond which the spots are consistently reduced to a single pixel.

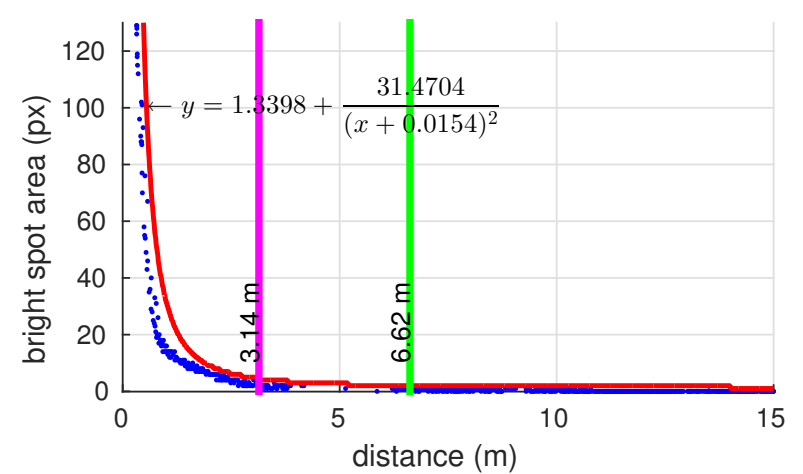

Fig. 11: The spot size to distance characteristic for the exposure rate of $1000 \mu \mathrm{s}$. The green line denotes the distance where the spot becomes a single pixel. The magenta line denotes the shortest distance, where spots with the size of $1 \mathrm{px}$ appear. The red curve is the approximating function of the maximum possible spot size.

in applications where very tight formations of micro aerial vehicles are needed we can select short exposure rate to reach a higher precision in distance estimation at short distances, in addition to better selectivity w.r.t. other light sources.

If the margins used in a swarming algorithm are derived from the spot sizes, then we can expand or shrink a formation simply by changing the exposure rate. This can even be done dynamically based on the circumstances, altering the swarm parameters on-the-fly (for instance by changing the constants in a Boids model [27] we can change the swarm size and thus adjust the working area).

Using a high exposition rate presents a trade-off, since it allows for detection and quantitative distance estimation in larger mutual distances, but has the side-effect of lower selectivity w.r.t. other light sources and thus lower robustness.

\section{B. Bearing vector estimation precision}

In order to verify the precision of the relative bearing vector estimation and the distance estimation based on the mutual distance of two spots in an image, we have equipped a hexarotor frame with an arm length of $0.4 \mathrm{~m}$ with UV light sources on the end of each arm. Precision and relia-

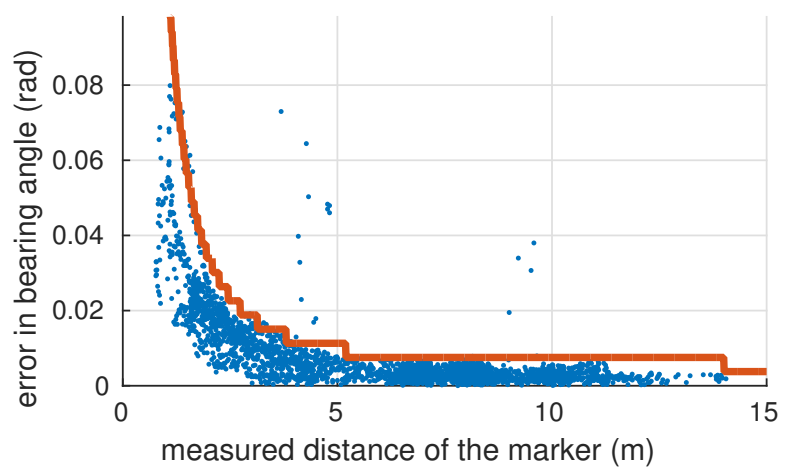

Fig. 12: Angles between the estimated bearing vectors based on the image and the true bearing vectors. The red line denotes the expected maximal errors based on equation 3

bility of the mutual distance estimation were evaluated in different relative positions and orientations using Optitrack as a ground truth.

Lines connecting the camera with the potentially visible markers were calculated from the ground truth poses of the camera and the hexarotor frame. Relative bearing vectors were estimated from the bright spots in the image. The bearing vectors and connecting lines were compared and the angles between the closest found matches were stored. Figure 12 shows, that the predicted maximal error in the estimation according to equation (3) holds.

\section{Neighbor distance from mutual distance of points}

In the same dataset as used previously, the pair of adjacent spots with the greatest mutual distance in the image was used as the basis for the distance estimation. The distance of the frame center from the camera was calculated both according to equation 5 and equation 6 . The estimated distances compared to the ground truth obtained from the OptiTrack system can be seen in figure 13, together with plots of the predicted margins of error based on the previously obtained function $S_{\max }(l)$. Note, that in the first case most estimates are greater than the ground-truth, while in the other case the opposite is true.

Figure 14 shows the relative errors in estimations in both cases.

Within the tested range the error increases roughly linearly with the distance. This is conforms to the expectations and is of no concern in forming a swarm, since the precision is only low outside of collision range.

The precision is decreased by the randomized angle of the observed pair of markers so that the results would be consistent with real-world situations.

For comparison, the precision listed in [2] was measured in ideal laboratory conditions with all variables accounted for. In such unrealistic conditions our system will exhibit higher precision, due to the larger baseline.

\section{Outdoor experiments of mutual localization of two UAVs}

To verify the selectivity of the sensor, as well as the range and precision in an outdoor environment, which is the 

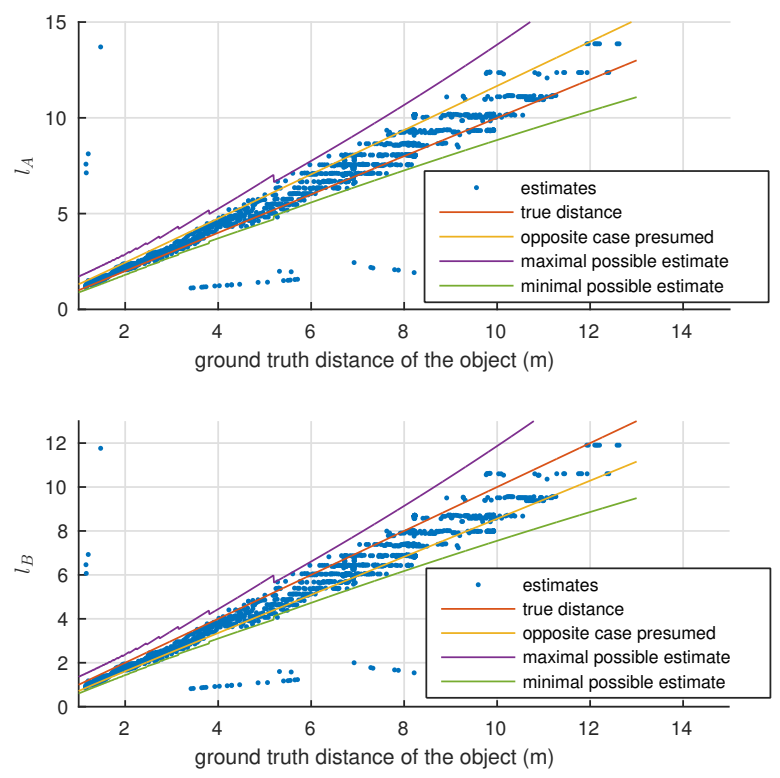

Fig. 13: The precision of estimation of the distance while presuming the orthogonal alignment (upper plot) and the $30^{\circ}$ alignment (lower plot) of the observed points.
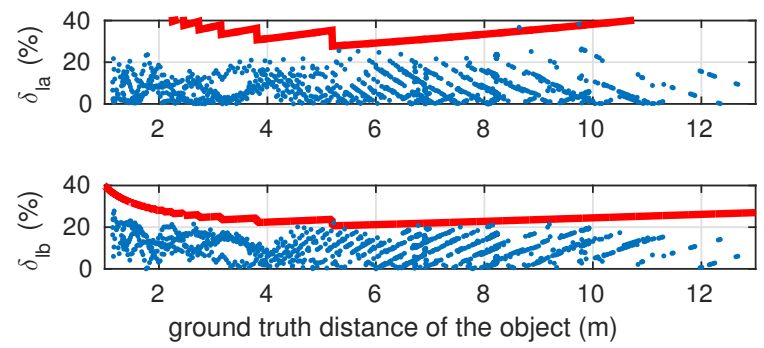

Fig. 14: Relative errors $\delta_{l a}$ and $\delta_{l b}$ of the estimated distances in case of presuming the orthogonal alignment (upper plot) and the $30^{\circ}$ alignment (lower plot) respectively. The red line denotes predicted maximal relative error.

primary intended use-case, we have performed a flight with a pair of our outdoor UAV platforms. The platform itself is a DJI 550 frame, equipped with a Pixhawk controller an Intel NUC computer, described in detail in [26]. The sensory equipment comprises a range sensor for altitude measurements, RTK-GNSS antenna for ground truth measurement, the UV camera described in this paper and a color camera for a conventional video recording. The target UAV was equipped with six ultraviolet LED markers as described above. The exposure rate of the camera was set to $1000 \mu \mathrm{s}$.

The markers could still be detected at $15 \mathrm{~m}$ and after that there were spurious drop-offs. Compare the table III in [2], where the maximum range is $5.5 \mathrm{~m}$ for the highest resolution. Indeed, since the camera FoV listed in the paper is only $42^{\circ}$ while our system uses a FoV of $180^{\circ}$, our system can be meaningfully compared with their system being used with less than the smallest listed resolution, where the maximum range was only $3.2 \mathrm{~m}$.

An example of the image obtained by the ultraviolet-

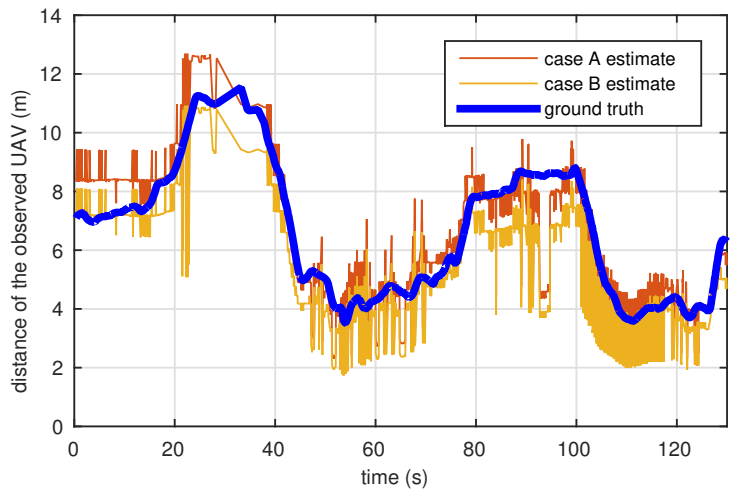

Fig. 15: UAV distance estimates compared with the ground truth. The two estimates according to equations (5) - case A and (6) - case B tend to be greater and smaller than the ground truth respectively. The spurious pattern around $60 \mathrm{~s}$ and $110 \mathrm{~s}$ is the result of the observed UAV spinning in place, leading to the two equations rapidly exchanging their validity.

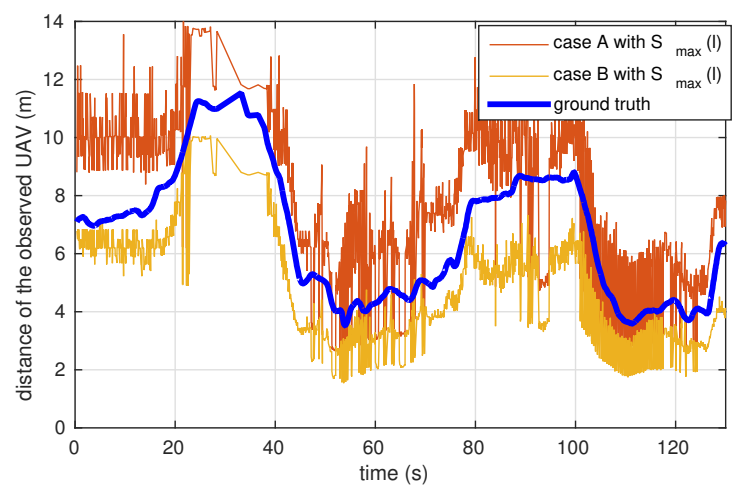

Fig. 16: The same estimates as in figure 15 with the angle $\alpha$ expanded by $k\left(S(l)-\frac{1}{2}\right)$.

sensitive camera can be seen in the figure 2. We have attempted to measure the precision of the ultraviolet markerbased localization by using the RTK-GNSS data inertial measurement unit and built-in compass as ground truth. As is shown in figures 15 and 16 , the distance estimations according to equations (5) and (6 resulted in upper and lower margins, enclosing the ground truth. While the position retrieved by RTK-GNSS was sufficiently precise, the orientation estimate was burdened by a severe drift (see figure 17below), causing misalignment in the bearing vector returned by our UV sensor. This hints at an alternative application of our system as a precise orientation sensor if the position of two or more mutually unoccluded UAVs is known from RTK-GNSS data, since the observed relative bearing does not drift.

\section{CONCLUSION}

In this paper we have proposed a novel system for outdoor and indoor mutual relative localization using ultraviolet LED makers. The main intended use-case of this method is in swarm control and stabilization of formations of light-weight helicopter UAVs in arbitrary environments. The proposed 


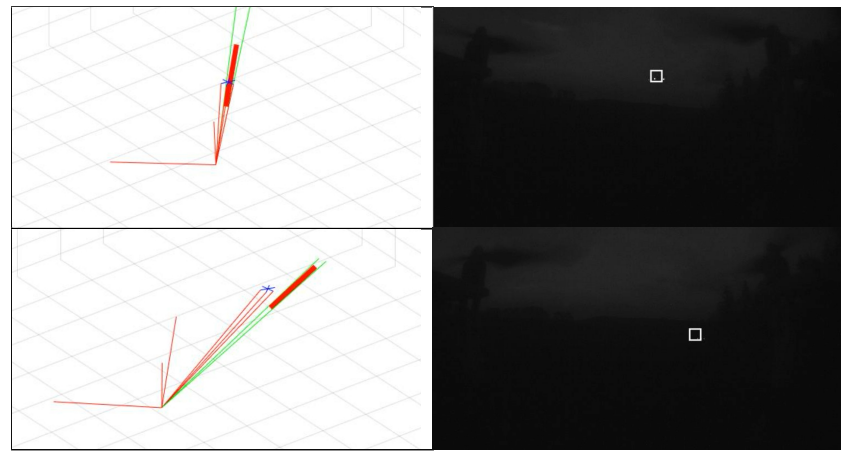

Fig. 17: Selected frames from the video output of the camera and visualization of the mutual state estimation procedure. The blue star and white square represent the ground truth of the observed UAV. The thick red line shows the range of possible positions computed by our system. This can be seen in a video found at http:// mrs.felk. cvut.cz/uvddl.

approach enables significantly higher detection range, robustness to light conditions and surrounding environment (background of images) in comparison with state-of-the-art methods, while having low computational intensity, small size and weight and providing sufficient precision. The error in the relative bearing vector towards a single ultraviolet marker is in operational distances below $0.02 \mathrm{rad}$ in distances above $3 \mathrm{~m}$, and thus allows direct applicability of the method using most of the current swarming and formation stabilization approaches. Two algorithms for estimating the mutual distance were developed to satisfy requirements of known multi-UAV stabilization techniques. The first relies on the size of the detected spot in the image, while the second is based on the diminishing apparent internal distance between a pair of retreating markers with known true mutual distance. The system was tested in outdoor environment and was shown to be robust with respect to outdoor lighting conditions as predicted. The theoretical predictions, as well as the experimental data presented here, show a lot of promise for deployment in swarm robotics.

\section{REFERENCES}

[1] M. Nagy, Z. Akos, D. Biro, and T. Vicsek, "Hierarchical group dynamics in pigeon flocks," Nature, vol. 464, no. 7290, p. 890, 2010.

[2] J. Faigl, T. Krajník, J. Chudoba, L. Preucil, and M. Saska, "Low-cost embedded system for relative localization in robotic swarms," in IEEE ICRA, 2013

[3] T. Krajník, M. Nitsche, J. Faigl, P. Vaněk, M. Saska, L. Přeučil, T. Duckett, and M. Mejail, "A practical multirobot localization system," Journal of Intelligent \& Robotic Systems, vol. 76, no. 3-4, pp. 539-562, 2014.

[4] M. Saska, V. Vonasek, T. Krajnik, and L. Preucil, "Coordination and Navigation of Heterogeneous UAVs-UGVs Teams Localized by a Hawk-Eye Approach," International Journal of Robotics Research, vol. 33, no. 10, pp. 1393-1412, 2014.

[5] M. Saska, T. Báča, J. Thomas, J. Chudoba, L. Preucil, T. Krajník, J. Faigl, G. Loianno, and V. Kumar, "System for deployment of groups of unmanned micro aerial vehicles in gps-denied environments using onboard visual relative localization," Autonomous Robots, vol. 41, no. 4, pp. 919-944, 2017.

[6] M. Saska, "Mav-swarms: Unmanned aerial vehicles stabilized along a given path using onboard relative localization," in International Conference on Unmanned Aircraft Systems (ICUAS). IEEE, 2015.
[7] K. Chaudhary, M. Zhao, F. Shi, X. Chen, K. Okada, and M. Inaba, "Robust real-time visual tracking using dual-frame deep comparison network integrated with correlation filters," in 2017 IEEE/RSJ International Conference on Intelligent Robots and Systems (IROS), 2017.

[8] A. Kushleyev, D. Mellinger, C. Powers, and V. Kumar, "Towards a swarm of agile micro quadrotors," Autonomous Robots, vol. 35, no. 4 2013

[9] P. Stegagno, M. Cognetti, G. Oriolo, H. H. Bülthoff, and A. Franchi, "Ground and aerial mutual localization using anonymous relativebearing measurements," IEEE Transactions on Robotics, vol. 32, no. 5 , pp. 1133-1151, Oct 2016.

[10] G. Vásárhelyi, C. Virágh, G. Somorjai, N. Tarcai, T. Szörényi, T. Nepusz, and T. Vicsek, "Outdoor flocking and formation flight with autonomous aerial robots," in IEEE/RSJ International Conference on Intelligent Robots and Systems (IROS), 2014. IEEE, 2014.

[11] C. Virágh, G. Vásárhelyi, N. Tarcai, T. Szörényi, G. Somorjai, T. Nepusz, and T. Vicsek, "Flocking algorithm for autonomous flying robots," Bioinspiration \& biomimetics, vol. 9, no. 2, 2014.

[12] G. Michieletto, A. Cenedese, and A. Franchi, "Bearing rigidity theory in SE(3)," in 55th IEEE Conf. on Decision and Control, Las Vegas, NV, Dec. 2016, pp. 5950-5955.

[13] A. Zunino, M. Crocco, S. Martelli, A. Trucco, A. D. Bue, and V. Murino, "Seeing the sound: A new multimodal imaging device for computer vision," in IEEE ICCVW, 2015.

[14] X. Wang, Y. Sekercioglu, and T. Drummond, "Vision-based cooperative pose estimation for localization in multi-robot systems equipped with rgb-d cameras," Robotics, vol. 4, no. 4, p. 122, Dec 2014.

[15] I. Senthooran, J. C. Barca, and H. Chung, "A 3d line alignment method for loop closure and mutual localisation in limited resourced mavs," in ICARCV, 2016.

[16] K. Boudjit and C. Larbes, "Detection and target tracking with a quadrotor using fuzzy logic," in 8th International Conference on Modelling, Identification and Control (ICMIC), 2016.

[17] V. Dhiman, J. Ryde, and J. J. Corso, "Mutual localization: Two camera relative 6-dof pose estimation from reciprocal fiducial observation," in IEEE/RSJ International Conference on Intelligent Robots and Systems, 2013

[18] R. Tron, J. Thomas, G. Loianno, J. Polin, V. Kumar, and K. Daniilidis, "Vision-based formation control of aerial vehicles," in Robotics: Science and Systems, 2014.

[19] I. Rekleitis, P. Babin, A. DePriest, S. Das, O. Falardeau, O. Dugas, and P. Giguere, "Experiments in quadrotor formation flying using on-board relative localization (technical report)." [Online]. Available: http://www2.ift.ulaval.ca/ pgiguere/ papers/ARdroneCL_Workshop.2015.pdf

[20] A. Censi, J. Strubel, C. Brandli, T. Delbruck, and D. Scaramuzza, "Low-latency localization by active led markers tracking using a dynamic vision sensor," in IEEE/RSJ International Conference on Intelligent Robots and Systems, 2013.

[21] D. Brandtner and M. Saska, "Coherent swarming of unmanned micro aerial vehicles with minimum computational and communication requirements," in European Conference on Mobile Robots (ECMR), 2017.

[22] A. Franchi, C. Masone, V. Grabe, M. Ryll, H. H. Bülthoff, and P. R. Giordano, "Modeling and control of uav bearing formations with bilateral high-level steering," The International Journal of Robotics Research, vol. 31, no. 12, 2012.

[23] D. Scaramuzza, A. Martinelli, and R. Siegwart, "A flexible technique for accurate omnidirectional camera calibration and structure from motion," in Fourth IEEE International Conference on Computer Vision Systems (ICVS'06), 2006.

[24] D. Scaramuzza. (2018) Ocamcalib: Omnidirectional camera calibration toolbox for matlab. Accessed on 02-18-2018. [Online]. Available: https://sites.google.com/site/scarabotix/ocamcalib-toolbox

[25] P. Stegagno, M. Cognetti, A. Franchi, and G. Oriolo, "Mutual localization using anonymous bearing measurements," in IEEE/RSJ International Conference on Intelligent Robots and Systems, 2011.

[26] G. Loianno, V. Spurny, T. Baca, J. Thomas, D. Thakur, D. Hert, R. Penicka, T. Krajnik, A. Zhou, A. Cho, M. Saska, and V. Kumar, "Localization, grasping, and transportation of magnetic objects by a team of mavs in challenging desert like environments," IEEE Robotics and Automation Letters, 2018, (accepted to RA-L and ICRA). [Online]. Available: http://ieeexplore.ieee.org/document/8276269/

[27] M. Saska, J. Vakula, and L. Preucil, "Swarms of Micro Aerial Vehicles Stabilized Under a Visual Relative Localization," in IEEE International Conference on Robotics and Automation (ICRA), 2014. 